\title{
Editorial
}

\section{Imaging, Vision, and Pattern Recognition}

\author{
Mourad Zaied $\left(\mathbb{D},{ }^{1}\right.$ Imed Bouchrika, ${ }^{2}$ Anil Kumar, ${ }^{3}$ Fouad Slimane, ${ }^{4}$ and Ridha Ejbali $\mathbb{D}^{5}$ \\ ${ }^{1}$ University of Gabès, Gabès, Tunisia \\ ${ }^{2}$ University of Souk Ahras Mohamed Chérif Messaadia, Souk Ahras, Algeria \\ ${ }^{3}$ Indian Institute of Information Technology, Design and Manufacturing Jabalpur, Jabalpur, India \\ ${ }^{4}$ Swiss Federal Institute of Technology in Lausanne, Lausanne, Switzerland \\ ${ }^{5}$ Faculty of Sciences of Gabès, Gabès, Tunisia \\ Correspondence should be addressed to Mourad Zaied; mourad.zaied@ieee.org
}

Received 15 April 2018; Accepted 15 April 2018; Published 3 May 2018

Copyright (C) 2018 Mourad Zaied et al. This is an open access article distributed under the Creative Commons Attribution License, which permits unrestricted use, distribution, and reproduction in any medium, provided the original work is properly cited.

Pattern recognition, machine vision, and imaging are a set of techniques and methods belonging to machine learning. They focus on approach linked to recognition of patterns, regularities in data, computer vision, and image processing. Pattern recognition, machine vision, and imaging share other topics such as artificial intelligence and learning techniques.

Moreover, signal processing is a fundamental domain based on several techniques which encompasses the fundamental theory, applications, algorithms, and implementation of processing or transferring information. It uses mathematical, statistical, and computational representations and formalisms for representation, modeling, analysis, and synthesis of data.

This special issue is dedicated to latest developments in the area of machine learning methods. The target audiences were researchers in machine learning and computational intelligence applied to image processing, signal processing, biomedical system, and security of the environment. After a strict review, seven articles from researchers around the world were finally accepted. In one paper, A. Helwan and D. U. Ozsahin proposed an approach based on a sliding window based machine learning system for the left ventricle localisation in MR cardiac images. In another paper, L. Duan et al. proposed a deep hashing based fusing index method for large-scale image retrieval. In one of the papers, H. Pan et al. proposed a regular $\mathrm{k}$-shrinkage thresholding operator for the removal of mixed Gaussian-impulse noise. In another paper, X. Sun et al. proposed a color image denoising based on guided filter and adaptive wavelet threshold. In one paper,
N. Nematzadeh and D. M. W. Powers proposed local and global perception from multiple scales to multiscale. Y. Liang et al. proposed multifeature and neighbor based salient region detection for social images. In another one, W. Chinsatit and T. Saitoh proposed CNN-based pupil center detection for wearable gaze estimation system.

\section{Acknowledgments}

We thank all the authors and reviewers for their great contributions to this special issue. We would also like to thank Professor Hsien-Chung Wu, the Editor in Chief, for his full support.

Mourad Zaied Imed Bouchrika Anil Kumar

Fouad Slimane Ridha Ejbali 


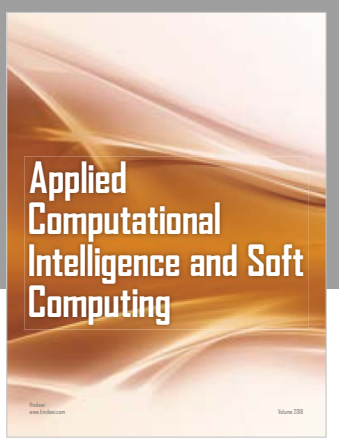

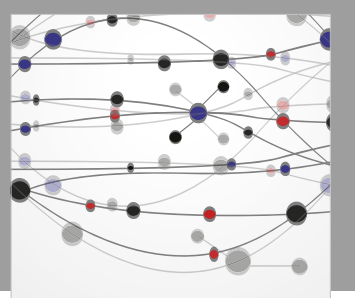

The Scientific World Journal
Submit your manuscripts at

Computing
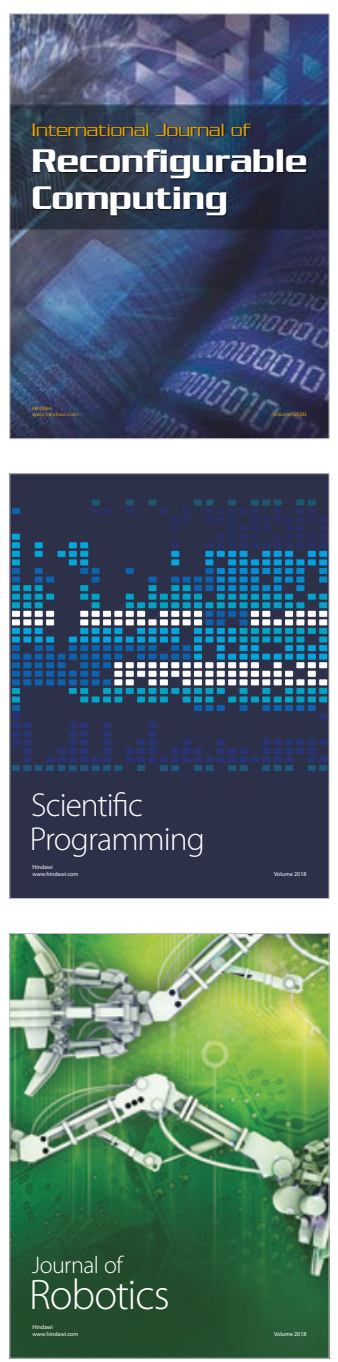

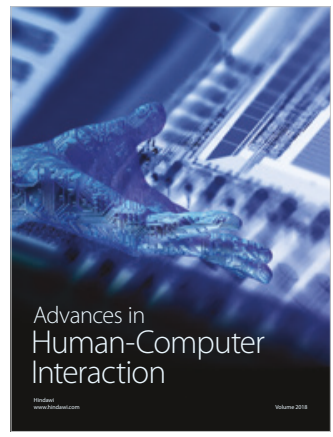

Human-Compute

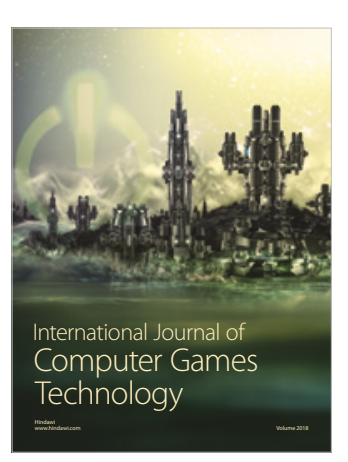

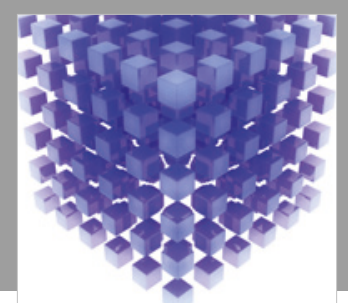

Mathematical Problems in Engineering

\section{Engincering}
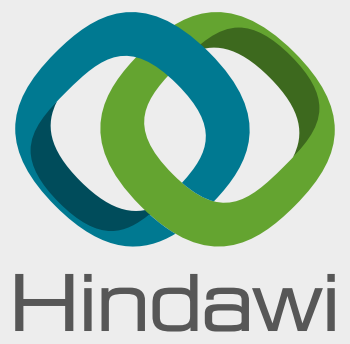

www.hindawi.com
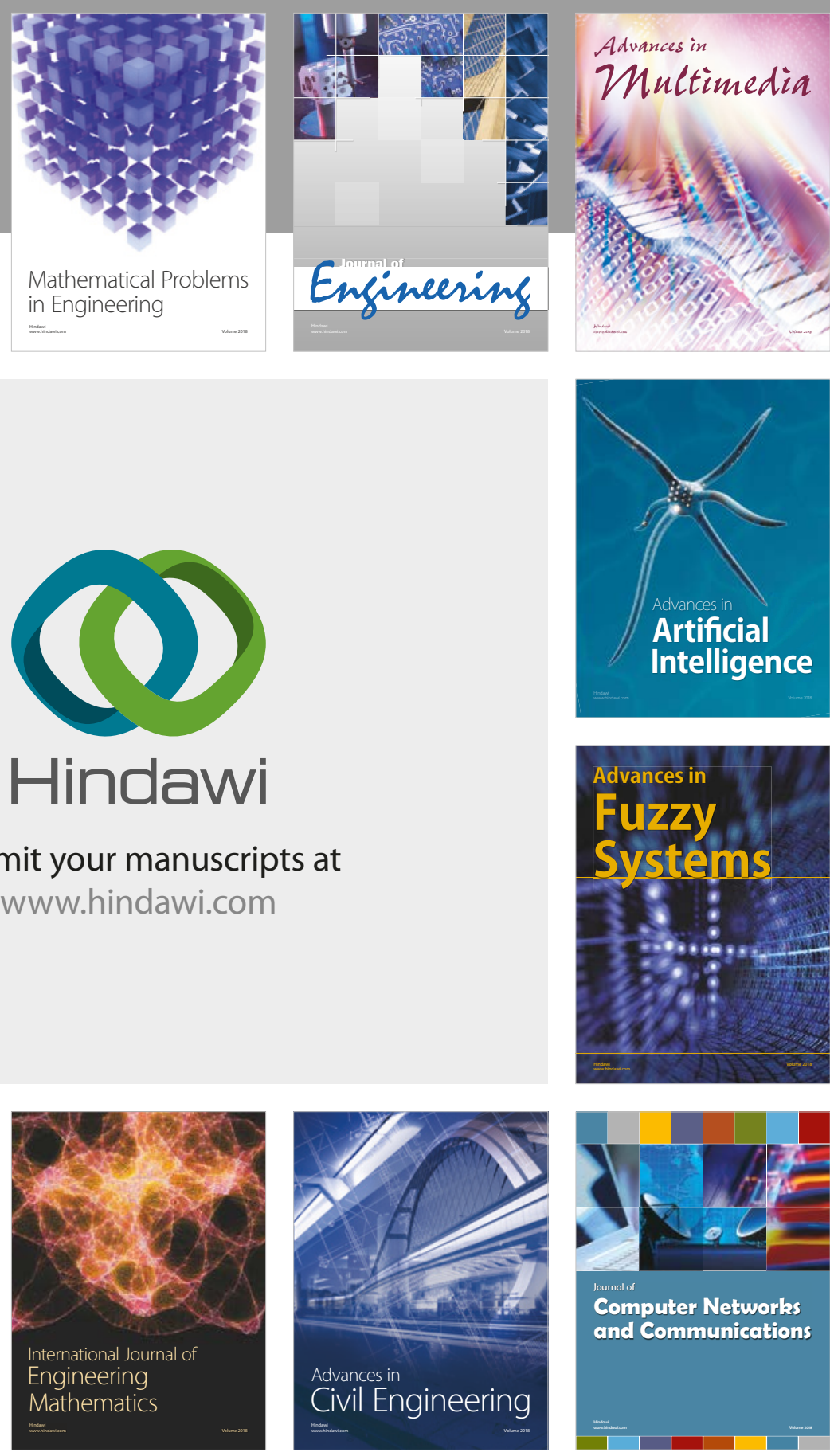

Computer Networks and Communications

Multimedia
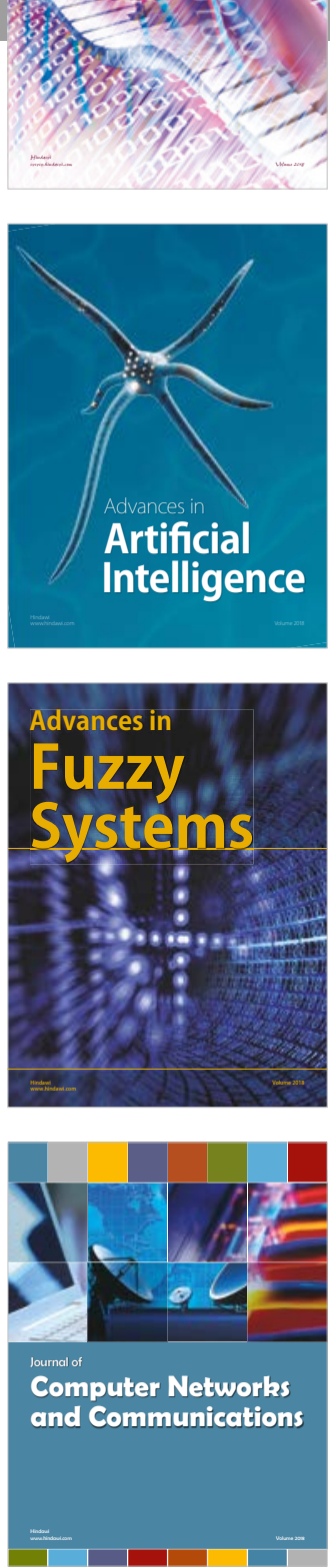

Advances in

Modelling \&

Simulation

in Engineering

interaction

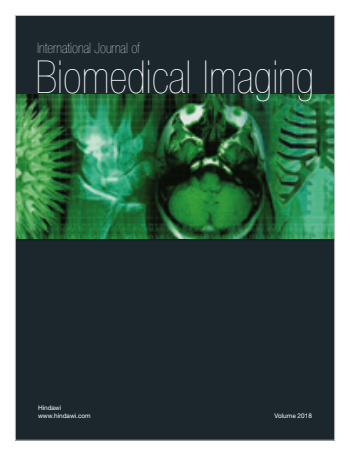

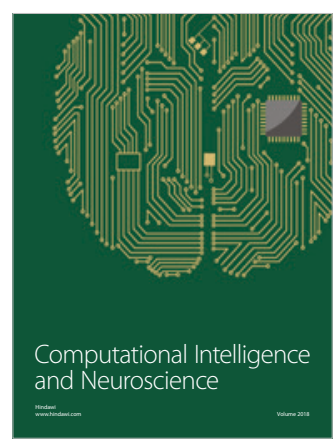

